\title{
Die EU und die Herausforderung der Migration: EU-Regionalpolitik als Lösungsansatz von Problemen?
}

\author{
Von Dimitrios Parashu, Hannover*
}

Der vorliegende Beitrag bietet zunächst einen kurzen Überblick zur Entwicklung der Europäischen Asyl- und Einwanderungspolitik über die letzten Dekaden, sowie eine synoptische Darstellung der (immer noch) aktuellen Flüchtlingskrise in der Union vor dem problematischen Hintergrund verschiedener Positionen der Mitgliedstaaten in diesem Kontext; der Beitrag mündet in Vorschläge de lege ferenda, deren erster allgemeinerer Natur ist und hierbei insbes. Elemente verstärkter Zusammenarbeit bemüht, und deren zweiter als dezidierter Lösungsansatz zur Einwanderungspolitik mit dem Ausgangspunkt der EU-Regionalpolitik, unter dem Einbezug institutionalisierter Ratsstrukturen, gedacht ist.

\section{Prolegomena}

Die letzte Dekade hat der EU eine - in dieser Diversität präzedenzlose - Sukzession von Krisen und Problematiken gebracht. Eines der zentralsten dieser Themen ist die Bewältigung der Flüchtlingskrise, welche die Union in verstärkter Intensität insbes. seit 2015 beschäftigt. An diesem Punkt, namentlich des sensiblen Topos der Aufnahme und Integration von Flüchtlingen drohen sich die Mitgliedstaaten heillos zu zerstreiten. Mithin stehen die Asyl- und die Einwanderungspolitik der EU auf dem Prüfstand.

Dies spürend, versucht die Union seit geraumer Zeit durch ihre legislativen Vorstöße und Aktionen eine Abhilfe zu schaffen; allerdings erscheint die Problematik bis dato nicht gelöst. Einige der Mitgliedstaaten möchten insbes. bei der Aufnahme und Integration dieser Menschen nicht teilnehmen, was wiederum diejenigen Mitgliedstaaten, welche aufgrund ihrer geographischen Lage zuerst mit Flüchtlingen aus Drittstaaten in Kontakt geraten, übermäßig belastet. Belastungen, welche die betreffenden Mitgliedstaaten (insbes. der Mittelmeerregion) kaum zu stemmen in der Lage sind.

Im Folgenden soll zunächst ein kurzer, zusammenfassender Überblick über die Entwicklung der Europäischen Asyl- und Einwanderungspolitik erfolgen (II). Daran schließt sich (III) die Formulierung von Lösungsgedanken an, welche (zunächst allgemeiner, III.1) als Ansatz für eine effektive Aufnahme und Integration von Migranten evtl. hilfreich sein könnten. Der abschließende Ansatz, konkreter hinsichtlich der Einwanderungspolitik (III.2), fußt auf einem Instrument, welches die EU ursprünglich eigens für die Harmonisierung von Unebenheiten zwischen

\footnotetext{
* Dr. iur., MLE, Dikigoros (griech. Rechtsanwalt), Habilitand und Wissenschaftlicher Mitarbeiter an der Juristischen Fakultät der Gottfried Wilhelm Leibniz Universität Hannover. Der Verf. dankt Herrn Prof. Dr. Armin Hatje für sehr wertvolle Bemerkungen und Anregungen.
} 
den Regionen ihrer Mitgliedstaaten entwickelte: Dies unter Einbezug institutionalisierter Ratsstrukturen.

\section{Hintergrund}

\section{Vom Dubliner Übereinkommen ${ }^{2}$ zur VO 604/2013³ (,Dublin III'-VO ${ }^{4}$ )}

Die Schaffung des Europäischen Binnenmarktes - mithin das sukzessive Zusammenwachsen der europäischen Wirtschaftselemente - haben den Topos der Zuwanderung in die heutige EU zu einem besonders diffizilen Sachgebiet avancieren lassen. In diesem Kontext ist bereits das Schengener Abkommen I aus dem Jahre 1985 als relevant zu nennen, obgleich dessen Spannbreite den Umfang der damaligen EG-Mitgliedstaaten schon übertraf: ${ }^{5}$ Durch dieses Abkommen wurden die innereuropäischen Grenzkontrollen zugunsten wirtschaftlicher Errungenschaften abgebaut, was freilich eigener, zusätzlicher Sicherheitsrisiken nicht entbehrte. Das Schengener Abkommen II aktualisierte im Jahre 1995 den Besitzstand aus Abkommen I. ${ }^{6}$

Sachverbunden, jedoch einige Jahre zuvor (konkret zwischen den beiden Schengener Abkommen) unterzeichneten alle damaligen zwölf Mitgliedstaaten der EG am 15. Juni 1990 ein Übereinkommen hinsichtlich der notwendigen Prüfung von Asylanträgen, ${ }^{7}$ welches gleichwohl (aufgrund der unterschiedlichen Ratifikationssituation in den bezeichneten Staaten) erst am 1. September 1997 in Kraft treten konnte. ${ }^{8}$ Dieses Dubliner Übereinkommen stellte namentlich auf zwei Ziele ab einerseits eine Verringerung des Arbeitsaufwandes national zuständiger Behörden

1 Siehe im Folgenden D. Parashu, IX. Elemente Europäischer Asylpolitik: Vom Dubliner Übereinkommen zur VO 604/2013 (,Dublin III'-VO), in: idem, Weiterführende Themen zur europäischen Rechtspraxis, 2018, 3. Aufl., S. $89 \mathrm{ff}$.

2 Übereinkommen über die Bestimmung des zuständigen Staates für die Prüfung eines in einem Mitgliedstaat der Europäischen Gemeinschaften gestellten Asylantrags, BGBl. 1994 II, S. 792/BGBl. 1997 II, S. 1452/ BGBl. 1998 II, S. 62. Vgl. auch B. Schattenberg, Deutsche Erfahrungen mit Schengen, in: Hailbronner/Weil (Hrsg.), Von Schengen nach Amsterdam: auf dem Weg zu einem europäischen Einwanderungs- und Asylrecht = From Schengen to Amsterdam (Schriftenreihe der Europäischen Rechtsakademie Trier, Bd. 29), 1999, S. 23, 27 f.; R. Fungueiriño-Lorenzo, Visa-, Asyl- und Einwanderungspolitik vor und nach dem Amsterdamer Vertrag. Entwicklung der gemeinschaftlichen Kompetenzen in: Visa-, Asyl- und Einwanderungspolitik (Europäische Hochschulschriften, Reihe II Rechtswissenschaft, Bd. 3527), 2002, 1. Aufl., S. 27 ff.; R. Weinzierl, Flüchtlinge: Schutz und Abwehr in der erweiterten EU. Funktionsweise, Folgen und Perspektiven der europäischen Integration (Schriftenreihe Europäisches Recht, Politik und Wirtschaft - hrsg. von Jürgen Schwarze - Bd. 308) 2005, S. 34-35

3 VO (EU) Nr. 604/2013 des Europäischen Parlaments und des Rates vom 26. Juni 2013 zur Festlegung der Kriterien und Verfahren zur Bestimmung des Mitgliedsstaats, der für die Prüfung eines von einem Drittstaatsangehörigen oder Staatenlosen in einem Mitgliedstaat gestellten Antrags auf internationalen Schutz zuständig ist (Neufassung); ABl. EU 2013 L 180/31. Vgl. hierzu etwa M. Herdegen, Europarecht, 2017, 19. Aufl., § 20, Rn. 3.

4 Vgl. zur Bezeichnung der VO etwa M. Herdegen (Fn. 3), § 20, Rn. 3.

5 Vgl. insbes. M. Herdegen (Fn. 3), § 20, Rn. 5 f.; R. Fungueiriño-Lorenzo (Fn. 2), S. 14 ff.; R. Weinzierl (Fn. 2), speziell S. $29 \mathrm{ff}$. zum ,Schengen-Besitzstand“ ferner auch $R$. Esser, Europäisches und Internationales Strafrecht, 2014, § 4, Rn. 1 ff.; B. Hecker, Europäisches Strafrecht, 2012, 4. Aufl., § 5, speziell Rn. 29 ff.

6 Ibid. Vgl. auch B. Schattenberg (Fn. 2), S. 23.

7 Siehe Fn. 2

8 Vgl. insbes. R. Weinzierl (Fn. 2), S. 34-35. 
durch die effektive Reduktion möglicher Mehrfachasylanträge und andererseits die Bekämpfung des durchweg problematischen Sachtopos der (von Staat zu Staat abgeschobenen) ,refugees in orbit' ${ }^{9}$ Die Kardinalsregelung des Übereinkommens stellte mithin die Bestimmung dar, dass ein Asylantrag nur in einem gemäß diesem Übereinkommen sachzuständigen Mitgliedstaat geprüft werden konnte (,One-State-Only'-Prinzip). ${ }^{10}$

Im Maastrichter Vertrag gehörte die Asylpolitik noch zur intergouvernementalen Säule der EU, folgerichtig war eine tiefergehende Verständigung zwischen den Mitgliedstaaten zur effektiven Lösungsfindung absolut vonnöten - durch den Vertrag von Amsterdam erfolgte die Überführung dieses Politikbereiches in den Kompetenzbereich der heutigen EU. ${ }^{11}$

Das bezeichnete Element der strikten Intergouvernementalität wurde recht bald überwunden - wohl, weil offensichtlich wurde, dass das Problem ein zu großes ist, um damit die Mitgliedstaaten allein zu lassen. Entsprechend wurde im EGV der Titel IV „Visa, Asyl, Einwanderung und andere Politiken betreffend den freien Personenverkehr" geschaffen. ${ }^{12}$

Zur besseren Institutionalisierung der Asylpolitik wurde in der Folge die VO ,Dublin II‘ (343/2003, ${ }^{13}$ geändert durch die VO (EG) 1103/2008 ${ }^{14}$ ) verabschiedet, welche sich insbesondere auch durch die Miteinbeziehung des damals neugeschaffenen elektronischen Identifizierungssystems ,EURODAC' auszeichnete. ${ }^{15}$ Auf der rein praktischen Ebene bot die VO 1560/2003 ${ }^{16}$ Durchführungsbestimmungen zu den allgemeineren Inhalten von Dublin II.

Als weiteres Anschauungsbeispiel für die nachfolgend intensivierte legislative Tätigkeit der EU auf dem bezeichneten Feld kann man hier ferner die Verabschie-

9 Vgl. R. Weinzierl (Fn. 2), S. 35.

10 Art. 3 Abs. 2 des Dubliner Übereinkommens; vgl. insbes. auch R. Weinzierl (Fn. 2), S. 35.

11 Vgl. etwa bereits M. Herdegen, Europarecht, 2008, 10. Aufl., § 21 I, Rn. 1/ § 31, Rn. 1 (unter Verweis auf $\S 14$ III ebendort); vgl. auch B. Schattenberg (Fn. 2), S.24/28-30; R. Fungueiriño-Lorenzo (Fn. 2), S. 68 ff.; $R$. Weinzierl (Fn. 2), S. 79 ff.

12 Ibid.

13 Verordnung (EG) Nr. 343/2003 des Rates vom 18. Februar 2003 zur Festlegung der Kriterien und Verfahren zur Bestimmung des Mitgliedstaats, der für die Prüfung eines von einem Drittstaatsangehörigen in einem Mitgliedstaat gestellten Asylantrags zuständig ist; ABl. EU 2003 L 50/1.

14 Verordnung (EG) Nr. 1103/2008 des Europäischen Parlaments und des Rates vom 22. Oktober 2008 zur Anpassung einiger Rechtsakte, für die das Verfahren des Artikels 251 des Vertrags gilt, an den Beschluss 1999/468/EG des Rates in Bezug auf das Regelungsverfahren mit Kontrolle; ABl. EU 2008 L 304/80.

15 Vgl. M. Herdegen (Fn. 3), § 20, Rn. 3; dort auch hinsichtlich der Verordnung (EG) Nr. 2725/2000 des Rates vom 11. Dezember 2000 über die Einrichtung von EURODAC für den Vergleich von Fingerabdrücken zum Zwecke der effektiven Anwendung des Dubliner Übereinkommens (ABl. EU 2000 L 316/1), welche bereits ersetzt wurde durch die Verordnung (EU) Nr.603/2013 des Europäischen Parlaments und des Rates vom 26. Juni 2013 über die Einrichtung von EURODAC für den Abgleich von Fingerabdruckdaten zum Zwecke der effektiven Anwendung der Verordnung (EU) Nr. 604/2013 (...), ABl. EU 2013 L 180/1. Zu besonderen Problematiken von EURODAC siehe etwa C. Gloor Scheidegger, Datenschutz und Rechtsschutz bei SIS, EURODAC und VIS, in: Breitenmoser/Gless/Lagodny (Hrsg.), Rechtsschutz bei Schengen und Dublin, 2013, S. 119 ff. Zum Status quo ante vgl. auch B. Schattenberg (Fn. 2), S. 28.

16 Verordnung (EG) Nr. 1560/2003 der Kommission vom 2. September 2003 mit Durchführungsbestimmungen zur Verordnung (EG) Nr. 343/2003 des Rates zur Festlegung der Kriterien und Verfahren zur Bestimmung des Mitgliedstaats, der für die Prüfung eines von einem Drittstaatsangehörigen in einem Mitgliedstaat gestellten Asylantrags zuständig ist; AB1. EU 2003 L 222/3. 
dung der verfahrensrechtlichen RL 2005/85/EG ${ }^{17}$ (reformiert durch die RL 2013/32/EU ${ }^{18}$ ) nennen. ${ }^{19}$

Aktuelle Legitimationsbasis der Union im Bereich der Asyl- und Einwanderungspolitik sind - neben dem Art. 3 Abs. 2 EUV - die Art. 78 und 79 AEUV. ${ }^{20}$ In Art. 78 ist festgelegt, dass sich die Mitgliedstaaten überhaupt eine Asylpolitik geben, unter Einbezug der ebenfalls höchstsensiblen Felder subsidiären und vorübergehenden Schutzes, und mit dem ständigen Ziele eines angemessenen Status und der prinzipiellen Nicht-Zurückweisung von Angehörigen eines Drittstaates. ${ }^{21}$

\section{Die Europäische Einwanderungspolitik}

Eng mit dem Sachtopos verknüpft, allerdings im Vergleich zum Status quo ante (Art. $63 \mathrm{EGV}$ ) nunmehr von der Asylpolitik emanzipiert und ,verselbständigt ${ }^{\circ}, 22$ ist ferner die Einwanderungspolitik der Union, welche ihre legitimatorische Grundlage (neben dem abermals zu bemühenden Art. 3 Abs. 2 EUV und dem dort bezeichneten rechtlichen Raum etc.) in Art. 79 AEUV findet. In letztgenanntem Artikel ist davon die Rede, dass die Union ,eine gemeinsame Einwanderungspolitik (entwickelt)“, mit den zugehörigen Charakteristika ,wirksame(r) Steuerung der Migrationsströme', , angemessene(r) Behandlung von (sich rechtmäßig in einem Mitgliedsstaate aufhaltenden) Drittstaatsangehörigen“ sowie schließlich der a priori und a posteriori erfolgenden ,Bekämpfung (..) illegaler Einwanderung und Menschenhandel ${ }^{6}{ }^{23}$ Diese Prämissen selbst kategorisiert Rossi freilich als „für sich noch nicht kompetenzbegründend“24. Damit hat er vollkommen recht der Rechtsanwender bekommt eher den Eindruck, dass es sich bei dieser Bestimmung um ein ausformuliertes Politikziel handelt: Mehr noch, „,(sind) die inhaltlichen Anforderungen an diese Politik (..) ausgesprochen anspruchsvoll und dürfen nur als unverbindliche Zielfestsetzungen verstanden werden, soll die gemeinsame Einwanderungspolitik nicht an ihren eigenen Erwartungen scheitern“25.

Dem europäischen Gesetzgeber sind jedenfalls, um diese Ziele verwirklichen zu können, konkrete Mittel vorbestimmt worden. So können Rat und Parlament im Rahmen des ordentlichen Gesetzgebungsverfahrens Maßnahmen verabschieden, welche sich namentlich auf die Unterbereiche ,Einreise- und Aufenthaltsvoraus-

17 Richtlinie 2005/85/EG des Rates vom 1. Dezember 2005 über Mindestnormen für Verfahren in den Mitgliedstaaten zur Zuerkennung und Aberkennung der Flüchtlingseigenschaft; AB1. EU 2005 L 326/13.

18 Richtlinie 2013/32/EU des Europäischen Parlaments und des Rates vom 26. Juni 2013 zu gemeinsamen Verfahren für die Zuerkennung und Aberkennung des internationalen Schutzes (Neufassung); AB1. EU 2013 L $180 / 60$.

19 Vgl. M. Herdegen (Fn. 3), § 20, Rn. 3.

20 Vgl. M. Herdegen (Fn. 3), § 20, Rn. 1/3.

21 Art. 78 Abs. 1 Satz 1 AEUV. Zur tiefergehenden Analyse siehe M. Rossi, in: Calliess/Ruffert, EUV/AEUVKommentar, 2016, 5. Aufl., Art. 78 AEUV, Rn. 1 ff.

22 M. Rossi, in: Calliess/Ruffert (Fn. 21), Art. 79 AEUV, Rn. 1.

23 Art. 79 Abs. 1 AEUV. Vgl. insbes. M. Herdegen (Fn. 3), § 20, Rn. 4.

24 So M. Rossi, in: Calliess/Ruffert (Fn. 21), Art. 79 AEUV, Rn. 2.

25 So M. Rossi, in: Calliess/Ruffert (Fn. 21), Art. 79 AEUV, Rn. 6. 
setzungen sowie Normen für die Erteilung von Visa und Aufenthaltstiteln', ,Festlegung (bestimmter) Rechte (von sich rechtmäßig in einem Mitgliedstaate aufhaltenden) Drittstaatsangehörigen', Prophylaxe ,illegaler Einwanderung und (entsprechenden) Aufenthaltes ${ }^{6}$, ferner der ,Bekämpfung des Menschenhandels` kaprizieren. ${ }^{26}$ Mit ebendiesen Bestimmungen werden ergo ,die konkreten Kompetenztitel (...) der Union`27 im gegebenen Zusammenhang artikuliert.

Auf demselben ordentlichen Gesetzgebungsweg können Rat und Parlament darüber hinaus besondere Maßnahmen zugunsten der Unterstützung integrativer Vorstöße der Mitgliedstaaten bezüglich sich dort legitim aufhaltender Angehöriger eines Drittstaates verabschieden. ${ }^{28}$ Diese Maßnahmen sollen interessanterweise ,unter Ausschluss jeglicher Harmonisierung der (mitgliedstaatlichen) Rechtsvorschriften“" 29 getroffen werden. Gleichwohl bleibt es den Mitgliedsstaaten selbst unbenommen, die genaue Anzahl der in diese einreisenden Angehörigen eines Drittstaates zwecks Arbeitssuche zu bestimmen. ${ }^{30}$ Dies wird nicht durch das Erfordernis expliziter Solidarität unter den Mitgliedstaaten im Zusammenhang der hier bezeichneten Politik ${ }^{31}$ konterkariert. Im hiesigen Folgeteil III.2 wird an diesen Punkt angeknüpft werden.

\section{Zentrales zu Dublin III}

Gegenstand der VO ist bereits fundamental die Festlegung konkreter Kriterien und entsprechender Verfahren zwecks positiver Bestimmung eines zur Prüfung eines Asylantragsgesuches zuständigen Mitgliedstaates. ${ }^{32}$ Um dieser Prämisse dienlich zu sein, hat der europäische Gesetzgeber einen sehr umfangreichen Definitionskatalog in die VO 604/2013 mitaufgenommen, ${ }^{33}$ welcher sachbezogene Termini wie etwa ,Drittstaatsangehöriger ${ }^{634}$ und ,Antrag auf internationalen Schutz $^{635}$ inhaltlich abschließend formuliert. Immens wichtig ist im gegebenen Zusammenhang ferner die - inhaltlich recht umfassende - Legaldefinition der relevanten ,Familienangehörigen ${ }^{\text {‘36. }}$.

Ein Herzstück der VO 604/2013 ist ganz unzweifelhaft der direkt darauffolgende Regelungskomplex bezüglich ,allgemeiner Grundsätze und Schutzgarantien “ der jeweils antragstellenden Personen. ${ }^{37}$ In diesem Duktus ist bereits die Pflicht der „Mitgliedstaaten, jeden Antrag auf internationalen Schutz (seitens eines) Dritt-

26 Art. 79 Abs. 2 AEUV.

27 So M. Rossi, in: Calliess/Ruffert (Fn. 21), Art. 79 AEUV, Rn. 2.

28 Art. 79 Abs. 4 AEUV.

29 Ibid.

30 Art. 79 Abs. 5 AEUV. Vgl. insbes. M. Herdegen (Fn. 3), § 20, Rn. 4.

31 Art. 80 AEUV. Vgl. insbes. M. Herdegen (Fn. 3), § 20, Rn. 4.

32 Art. 1 VO 604/2013.

33 Art. 2 VO 604/2013.

34 Art. 2 lit. a VO 604/2013.

35 Art. 2 lit. b VO 604/2013.

36 Art. 2 lit. g VO 604/2013.

37 Art. 3 ff. VO 604/2013. 
staatsangehörige(n) oder Staatenlose(n) (zu prüfen)“38 als charakteristisch für die hiesige Intention des Gesetzgebers auszumachen, schon aus rechtsstaatlichem Gesichtspunkt betrachtet keinen Antrag effektiv unbeantwortet zu lassen. Diese Prüfung kann nur durch einen letztlich zuständigen Mitgliedstaat vorgenommen werden; ${ }^{39}$ ultimativ prüfungszuständig ist der Staat, an welchen zuerst ein Schutzgesuch gerichtet worden ist. ${ }^{40}$ Im Rahmen des Prüfungsverfahrens ist dem Interessierten ein umfangreiches ,Recht auf Information“41 einzuräumen, und auch dem für die Ermittlung des prüfungszuständigen Staates relevanten ,persönliche(n) Gespräch ${ }^{42}$ mit dem Gesuchenden sowie bestimmten und absolut notwendigen ,Garantien für Minderjährige ${ }^{43}$ ist jedenfalls starke Bedeutung beizumessen.

Zweites und wohl noch maßgeblicheres Kernelement der VO 604/2013 sind die Art. 7 ff. derselben, in welchen die Kriterien zur Bestimmung des prüfungszuständigen Mitgliedstaates deutlich festgelegt werden. Die Rangfolge der Kriterien ist hierbei präzise festgelegt. ${ }^{44}$

Zunächst ist (im Falle minderjähriger Gesuchender ohne Begleitung) der Mitgliedstaat prüfungszuständig, in welchem ,sich ein Familienangehöriger oder eines der Geschwister des unbegleiteten Minderjährigen rechtmäßig aufhält “". ${ }^{45}$ Hierbei ist jedoch stets das Kindeswohl im Auge zu behalten. ${ }^{46}$ Bei verheirateten Minderjährigen ist der Mitgliedstaat zuständig, in welchem der Ehepartner sich legal aufhält; ${ }^{47}$ falls letzteres nicht der Fall sein sollte, greift wiederum die Staatszuständigkeit der engen Familienangehörigen nach Art. 8 Abs. 1 Satz 1 der VO 604/2013. ${ }^{48}$ In Ermangelung enger Familienangehöriger kommen die Mitgliedstaaten des Aufenthaltes entfernterer Verwandter, ${ }^{49}$ bei Aufenthalt der engen Familienangehörigen in mehreren denn einem Mitgliedstaate das effektive Kindeswohl zum Zuge. ${ }^{50}$ Die genannten Ergebnisse entstehen gleichsam, falls ein relevanter Familienangehöriger zugleich auch ,Begünstigter internationalen Schutzes“ ist $^{51}$ oder solches beantragt hat ${ }^{52}$. Auch müht sich die VO 604/2013 nach Kräften, Verfahren betreffend ganzer Familien in toto zu behandeln. ${ }^{53}$

Falls der Gesuchende in einem Staat einen gültigen Aufenthaltstitel erworben hat, ist derselbe Staat für die Prüfung des Gesuches zwecks internationalem Schutz

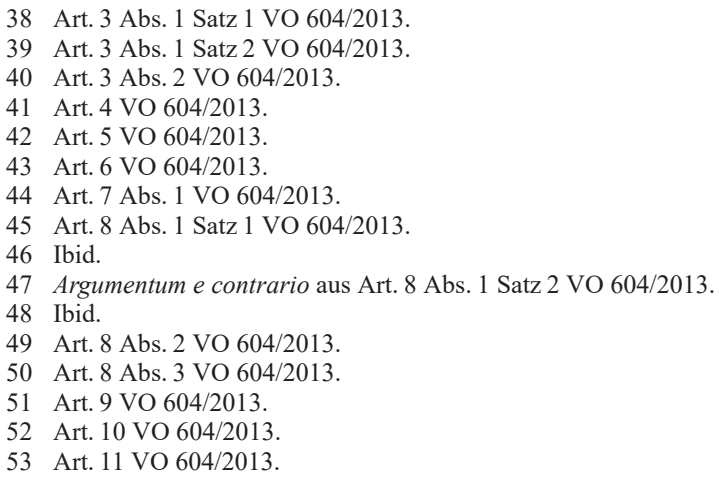


sachzuständig; 54 bei illegaler Einreise des Gesuchenden in einen Mitgliedstaat entsprechend letzterer. ${ }^{55}$ Die Prüfungszuständigkeit für ein Gesuch auf internationalen Schutz haben ferner Staaten ,ohne Visumszwang ${ }^{6}$ der betreffenden Person, ${ }^{56}$ und diejenigen Staaten, in deren Flughafen-Transitbereich eine betreffende Person ihr entsprechendes Gesuch stellt. ${ }^{57}$

Neben der soeben geschilderten Kriterienreihenfolge zur Ermittlung des gesuchsprüfungszuständigen Mitgliedstaates können freilich auch diverse Härtefallregelungen greifen, worunter explizit die Existenz abhängiger Personen als Antragsteller $^{58}$ sowie ,Ermessensklauseln ${ }^{659}$, gemäß welchen ein Mitgliedstaat ausnahmsweise die entsprechende Zuständigkeit zum Wohle der betreffenden Person an sich ziehen kann, ${ }^{60}$ fallen.

Die Art. 18 ff. der VO 604/2013 regeln darauffolgend die einschlägigen Pflichten des Mitgliedstaates, wessen Zuständigkeit auf Basis ebendieser VO festgestellt worden ist (Art. 18 VO 604/2013 - insbesondere die Aufnahme eines Antragstellers stellt hierbei eine Kardinalspflicht dar $)^{61}$; ferner die detaillierteren Modalitäten eines entsprechenden Aufnahme- respektive Wiederaufnahmeverfahrens (Art. 20 ff. VO 604/2013) sowie die Überstellung der interessierten Person in den letztlich zuständigen Mitgliedstaat (Art. 29 ff. VO 604/2013). Besonders bedeutsam ist in diesem Zusammenhang nicht zuletzt die Vertiefung einschlägiger Verfahrensgarantien, insbesondere dergestalt, dass eine Inhaftnahme des Gesuchenden die Erfüllung ganz bestimmter Voraussetzungen verlangt (Art. 28 VO 604/2013).

Die gegenseitige Information der Mitgliedstaaten ${ }^{62}$ in Verbindung mit einem ,Frühwarnmechanismus ${ }^{\text {63 }}$ gegenüber bestimmten asylrechtlich relevanten Krisenherden und einem potentiellen Verfahren zur Schlichtung einschlägiger (Zuständigkeits-)Konflikte zwischen Mitgliedstaaten ${ }^{64}$ runden allgemein den theoretischen Regelungsinhalt dieser VO ab. Die entsprechenden Kooperationselemente scheinen jedoch in den letzten Jahren Probleme aufzuweisen.

61 Art. 18 Abs. 1 lit. a VO 604/2013.

62 Art. 31 ff. / 34 ff. VO 604/2013. Vgl., hinsichtlich Finanzinformationen der Betreffenden, A. Lobsiger, Rechtsschutz beim amtshilfeweisen Austausch von Finanzinformationen, in: Breitenmoser/Gless/Lagodny (Hrsg.), Rechtsschutz bei Schengen und Dublin, 2013, S. $67 \mathrm{ff}$.

63 Art. 33 VO 604/2013.

64 Art. 37 VO 604/2013. 


\section{Würdigung und Ausblick: Die Herausforderung der aktuellen Flüchtlingskrise ${ }^{65}$}

Die EU sucht in den letzten Jahren, wie gesehen, verstärkt auch auf dem sensiblen Felde der Migrationsproblematik gesetzgeberisch tätig zu werden. Allerdings scheint all dies im Lichte jüngerer Ereignisse nicht auszureichen: Gemäß beispielsweise der hellenischen UNHCR-Sektion stieg die Zahl der illegalen Grenzübertritte in der Ostägäis bereits von Januar bis Ende August 2014 gegenüber dem entsprechenden Vorjahreszeitraum von 6.834 auf insgesamt bereits 22.089, also knapp das Vierfache. ${ }^{66}$ Diese Tendenz ist seitdem verstärkt - und die Beschaffenheit insbes. der hellenisch-türkischen Seegrenze im Ägäischen Meer ist hierbei freilich höchst charakteristisch und begünstigt diese Entwicklung.

Infolge der verstärkten Flüchtlingskrise des Sommers 2015 sieht sich das geschilderte ,Dublin-System ' mithin auf dem Prüfstand; die Kommission ist derzeit damit beschäftigt, eine entsprechend aktualisierte Strategie zu entwickeln. ${ }^{67}$ Die jüngsten Vorschläge umfassen weitergehende Harmonisierungen bzgl. der Aufnahmebedingungen und der Asylanerkennung, einen Neuansiedlungsrahmen und eine „vollwertige Asylagentur der Europäischen Union“. ${ }^{68}$ Daneben hat es aber in jüngerer Zeit verstärkt einschlägigen Streit zwischen der Union und Mitgliedstaaten gegeben. ${ }^{69} \mathrm{Ob}$ zeitweilige Maßnahmen, wie etwa ein sachbezogenes Abkommen der EU mit der Türkischen Republik vom März 2016, langfristig effektiv sein können, wird sich weisen.

65 Siehe im Folgenden D. Parashu, Der Grenzzaun zwischen der Hellenischen und der Türkischen Republik Analyse vor dem Hintergrund der Gemeinsamen Einwanderungspolitik der EU, in: Schneider/Wahl (Hrsg.), Herausforderungen für das Recht der zivilen Sicherheit in Europa. Aktuelle Beiträge des Kompetenznetzwerkes KORSE (Schriftenreihe „Sicherheit und Gesellschaft. Freiburger Studien des Centre for Security and Society“, hrsg. von Hans-Helmuth Gander/Walter Perron/Ralf Poscher/Gisela Riescher/ Thomas Würtenberger, Bd. 9), 2016, 1. Aufl., S. 137 ff. (145-147).

66 Siehe den Bericht (ohne Autorenangabe), UNO: Zahl der Flüchtlinge in Ägäis verdreifacht' auf orf.at vom 8. Oktober 2014, abrufbar unter http://orf.at/stories/2248832/ (zuletzt besucht am 22. November 2018).

67 Vgl. ,Migration und Asyl. Maßnahmen der Kommission', abrufbar unter https://ec.europa.eu/info/topics/migr ation-and-asylum de (zuletzt besucht am 22. November 2018). Vgl. ebenso „Eine umfassende Migrationssteuerung. Vermerk der Kommission zur Tagung des Europäischen Rates im Juni 2018“, abrufbar unter https:/ /ec.europa.eu/commission/sites/beta-political/files/euco-migration-booklet-june2018 de.pdf (zuletzt besucht am 22. November 2018).

68 Vgl. „Eine umfassende Migrationssteuerung. Vermerk der Kommission zur Tagung des Europäischen Rates im Juni 2018“ (Fn. 67), insbes. S. 5-6.

69 Vgl. ,Europäische Kommission - Pressemitteilung (vom 26. Juli 2017): Umverteilung: Kommission leitet nächste Stufe der Vertragsverletzungsverfahren gegen die Tschechische Republik, Ungarn und Polen ein', abrufbar unter http://europa.eu/rapid/press-release_IP-17-2103_de.htm (zuletzt besucht am 22. November 2018). 


\section{Vorschläge de lege ferenda}

\section{Allgemeiner Ansatz ${ }^{70}$}

Um der gesamten Problematik effektiver zu begegnen, erscheint es allerdings auch vonnöten, den Mitgliedstaaten entgegenzukommen. Bei deren Unwillen zur Partizipation könnten thematisch einschlägige Maßnahmen u.U. bereits auf Basis der Figur ,Verstärkter Zusammenarbeit ${ }^{671}$, ferner auch vermöge von ,opt-in“Strukturen (siehe hierzu rein exemplarisch den VO-Vorschlag zum Gemeinsamen Europäischen Kaufrecht) ${ }^{72}$ angedacht werden. So ließe sich die zwischenmitgliedstaatliche Zusammenarbeit vertiefen, um dergestalt auch dem Erfordernis von mitgliedstaatlicher Solidarität ${ }^{73}$ Genüge zu tun. Es erscheint diskutabel, ob dies im Lichte jüngerer Nachbesserungsansätze des europäischen Gesetzgebers letztlich schon erreichbar ist. ${ }^{74}$ Ferner könnte auf Basis der Gemeinsamen Nachbarschaftspolitik des Art. 8 EUV, welche sich insbes. auf Mittelmeer-Anrainerstaaten der EU kapriziert, ein völkerrechtliches Instrument geschaffen werden, welches kriminellen Schleuserbanden effektiver das Handwerk legen würde - unter Einbezug diverser, aber stets einheitlich konkret zu beziffernder Möglichkeiten finanzieller Sanktionen gegen zuwiderhandelnde Staaten.

\section{Lösungsansatz zur Einwanderungspolitik mit dem Ausgangspunkt der EU-Regionalpolitik}

Die Regionalpolitik der Europäischen Union, welche bereits auf eine Vielzahl erfolgreicher Strukturmaßnahmen verweisen kann, ${ }^{75}$ verfolgt (hier soll uns der status quo nach Lissabon beschäftigen) einen bestimmten Duktus ${ }^{76}$ : Elemente des „wirtschaftlichen, sozialen und territorialen Zusammenhaltes“ (bereits supra Art. 174 AEUV) sollen der Förderung der ,harmonische(n) Entwicklung der Union als Ganzes“"77 dienlich sein. In der weiteren Folge dieses primärrechtlichen Artikels wird evident, dass es hier zentral um eine Harmonisierung des Entwicklungsstandes potentiell zurückgebliebener Regionen der Mitgliedstaaten geht

70 Vgl. D. Parashu (Fn. 1), S. 98-99.

71 Siehe Art. 20 EUV / Art. 326-334 AEUV.

72 Vorschlag für eine Verordnung des Europäischen Parlaments und des Rates über ein Gemeinsames Europäisches Kaufrecht vom 11. Oktober 2011 (KOM (2011) 635 endg.).

73 Vgl. hier den Zusammenhang des Art. 80 AEUV.

74 VO (EU) Nr. 1168/2011 des Europäischen Parlaments und des Rates vom 25. Oktober 2011 zur Änderung der Verordnung (EG) Nr. 2007/2004 des Rates zur Errichtung einer Europäischen Agentur für die operative Zusammenarbeit an den Außengrenzen der Mitgliedstaaten der Europäischen Union, AB1. EU 2011 L 304/1; in gewissem Maße auch die VO (EU) Nr. 1052/2013 des Europäischen Parlaments und des Rates vom 22. Oktober 2013 zur Errichtung eines Europäischen Grenzüberwachungssystems (EUROSUR), AB1. EU 2013 L 295/11.

75 Vgl. exemplarisch bereits Europäische Union (Hrsg.), ,Regionalpolitik‘ vom 24. Mai 2018, abrufbar unter https://europa.eu/european-union/topics/regional-policy de (zuletzt besucht am 22. November 2018).

76 Art. 174-178 AEUV.

77 Siehe Art. 174 Satz 1 AEUV. 
(Art. 174 Satz 2 AEUV), ja dies insbes. ländliche sowie Gebiete industriellen Wandels betrifft, wobei Fragen natürlicher und demographischer Probleme besonderes Augenmerk erfahren (Art. 174 Satz 3 AEUV).

Dementsprechend ist die Umsetzung dieser Politik ganz eng mit der mitgliedstaatlichen Wirtschaftspolitik verbunden, ${ }^{78}$ unter einschlägiger Unterstützung insbes. seitens der Unionsstrukturfonds. ${ }^{79} \mathrm{Zu}$ letzteren gehören ex lege der Europäische Ausrichtungs- und Garantiefonds für die Landwirtschaft - Abteilung Ausrichtung, der Europäische Sozialfonds und der Europäische Fonds für regionale Entwicklung. ${ }^{80}$

Daneben hat das Primärrecht allerdings auch vorgesehen, dass vermöge einer potentiellen Erforderlichkeit „,spezifischer Aktionen außerhalb der Fonds" ${ }^{\text {"81, }}$, der ordentliche EU-Gesetzgebungsweg gegeben ist. ${ }^{82}$ Dies gilt selbstredend auch für die Strukturfonds insgesamt und deren mögliche Modifikationsbedürfnisse. ${ }^{83}$ Es steht die Frage im Raum, ob eine solche Erforderlichkeit angesichts der Flüchtlingskrise der letzten Jahre gegeben wäre.

Da die bestmögliche Integration von legal in die Union gekommenen Drittstaatsangehörigen ganz wesentlich auch den wirtschaftlichen und sozialen Zusammenhalt innerhalb der Union, wie Art. 174 AEUV diesen anführt, betrifft, stellt sich daneben auch die Frage, ob eine etwa gezielte Modifikation respektive Erweiterung der Strukturfonds hier einen Lösungsansatz bieten könnte. Dieser Ansatz soll an dieser Stelle, aus Gründen institutionellen Gleichgewichts, wie folgt aufgebaut sein.

Das EU-Primärrecht sieht vor, dass der Ratsvorsitz auf Basis eines ,System(s) der gleichberechtigten Rotation" durchgeführt wird (so Art. 16 Abs. 9 EUV). ${ }^{84}$ Zum Zwecke der Steigerung der operativen Handlungsfähigkeit ${ }^{85}$ besteht seit Lissabon die sogenannte ,Teampräsidentschaft ${ }^{\star 86}$, „welche sich aus jeweils drei Mitglied-

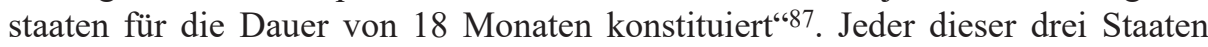
übernimmt in diesem Rahmen sechs Monate lang den Vorsitz in allen Ratskonstellationen (gem. Art. 16 Abs. 9 EUV exklusive des Rates für Auswärtige Angelegenheiten). ${ }^{88}$ Eine rudimentäre Fluktuation ist in gewissem Maße evident, soll aber durch bestimmte, hier sogleich anzusprechende Charakteristika des Ratsvorsitzes ausgeräumt werden.

Calliess räumte hierbei ein, dass „die Regelung des Vorsitzes (...) eine nahezu unauflösbare Gratwanderung zwischen Kontinuität und Effizienz einerseits und

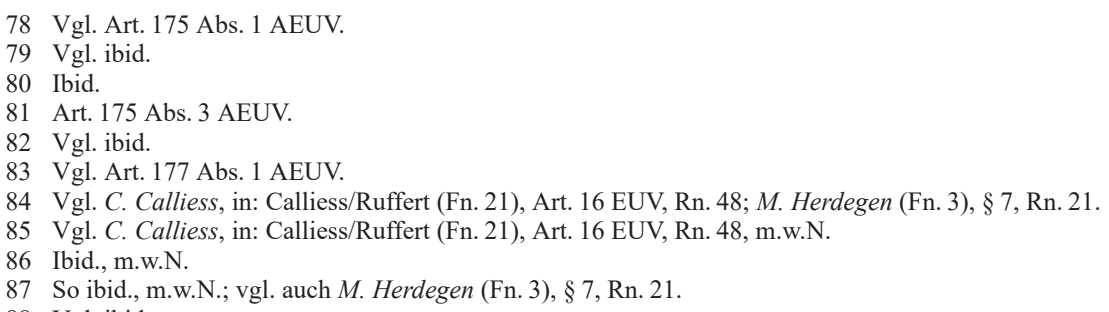


der gleichberechtigten Teilhabe aller Mitgliedstaaten an Führungsfunktionen im Rat sowie der Vermeidung einzelstaatlicher Machtkonzentrationen andererseits (ist)“89. Auf Basis des hier nachfolgenden Vorschlags könnte diese ,Gratwanderung' dazu verwendet werden, den Widerwillen, den einige Mitgliedstaaten bei einer Kooperation im Rahmen der Integration von Flüchtlingen dokumentieren, etwas zu kanalisieren. Denn eine solch ,gleichberechtigte Teilhabe an Führungspositionen' würde freilich auch bestimmte, institutionalisierte Verantwortung nach sich ziehen.

Dem steht nicht entgegen, dass der Ratsvorsitz selbst ex lege keine Kompetenzen hat; ${ }^{90}$ er füllt allerdings Funktionen innerhalb des Rates aus. ${ }^{91}$ Eine solche Funktion würde auch die hier angedachte Rolle einer zentralen Figur im Angesicht von Kalamitäten der EU-Migrationspolitik ausfüllen. Denn bereits gemäß der Geschäftsordnung des Rates ${ }^{92}$ (vgl. insbes. deren Art. 20) ist dem Vorsitz etwa die interne Organisation der Ratsarbeit anheimgestellt. ${ }^{93}$ Doch konkreter:

Die Mitgliedstaaten, welche im Rahmen Ihrer Ratspräsidentschaften einen solchen ,Dreiervorsitz ${ }^{94}$ bilden, könnten aufgrund dieser besonderen institutionellen Verantwortung eine modifizierte oder spezielle Strukturfondsfinanzierung erhalten, mit dem Ziel, legal in die EU gekommene Drittstaatsangehörige entsprechend den Möglichkeiten ihrer Regionen aus-, respektive weiterzubilden (die entsprechende Abfolge der Ratspräsidentschaften in der unmittelbaren Zukunft sieht zunächst, ab 2019, Rumänien/Finnland/Kroatien vor; ab dem 2. Halbjahr 2020 bis Ende des Jahres 2021 Deutschland/Portugal/Slowenien, etc. ${ }^{95}$. Anhand der bis zu einem Stichtag zu übermittelnden einschlägigen Bedürfnisse des jeweiligen nationalen Arbeitsmarktes bestünde die Möglichkeit, diese entsprechend aus- oder weitergebildeten Personen sodann, gemäß der Reihenfolge der Staaten bei den Ratspräsidentschaften bis 2030, in den Mitgliedstaaten gezielt integrieren zu können. Die Anknüpfung an den Ratsvorsitz erscheint deswegen als passend, weil im Rahmen des ,Dreiervorsitzes“ regelmäßig Mitgliedstaaten mit ganz unterschiedlichen Infrastrukturen dazu bestimmt sind, zu interagieren. Auf diese Art und Weise wäre es evtl. möglich, verschiedentlich bestehenden Dissens zwischen Mitgliedstaaten in kleinschrittiger Form zu überwinden. Die Anbindung des Ganzen an den ,Dreiervorsitz w würde aufgrund dessen Organisationsform die Gewähr dafür bieten, dass kein Mitgliedstaat diesbezüglich übervorteilt oder benachteiligt würde: Eine Interaktion , a trois` zugunsten gemeinschaftlichen Fortschrittes.

89 So C. Calliess, in: Calliess/Ruffert (Fn. 21), Art. 16 EUV, Rn. 49.

90 Vgl. C. Calliess, in: Calliess/Ruffert (Fn. 21), Art. 16 EUV, Rn. 50.

91 Vgl. ibid., m.w.N.

92 Vgl. hierzu die Verordnung 2009/937/EU des Rates vom 1. Dezember 2009 zur Änderung seiner Geschäftsordnung (AB1. EU 2009, L 325/35).

93 Vgl. C. Calliess, in: Calliess/Ruffert (Fn. 21), Art. 16 EUV, Rn. 50.

94 Vgl. bereits Art. 1 Abs. 1 und siehe insbes. den Anhang I des Beschlusses (EU) 2016/1316 des Rates vom 26. Juli 2016 zur Änderung des Beschlusses 2009/908/EU zur Festlegung von Maßnahmen für die Durchführung des Beschlusses des Europäischen Rates über die Ausübung des Vorsitzes im Rat und über den Vorsitz in den Vorbereitungsgremien des Rates, ABl. EU 2016 L 208/42.

95 Vgl. Anhang I zum Beschluss (EU) 2016/1316. 
Eine bloße Begünstigung (mit Strukturfondsmitteln) aller Mitgliedstaaten, die sich um die Weiter- und Ausbildung von Zugewanderten bemühten, hätte m.E. nicht den Vorteil, dass sich jeweils drei - unterschiedlich strukturierte - Mitgliedstaaten institutionell mit der Problematik auseinandersetzen müssten (wobei sukzessive alle Mitgliedstaaten an die Reihe kämen).

Ex lege spräche ein solches Vorgehen nicht gegen die Haushaltskompetenz der Kommission (initiativ) wie auch des Rates und des Parlaments bereits aus Art. 314 AEUV. ${ }^{96}$ Die Kommission ist bekanntlich maßgeblich der Förderung der Unionsinteressen verschrieben, ${ }^{97}$ insoweit wäre die Finanzierung eines Lösungsansatzes der Migrationsproblematik auf der hier vorgeschlagenen Ebene, welcher angesichts beschriebener Kalamitäten gewiss im Interesse der Weiterentwicklung der Union wäre, durch das Primärrecht gedeckt. Die Kommission hat gemäß Art. 314 Abs. 5 AEUV darüber hinaus auch die Möglichkeit, schlichtend im Falle von etwa divergenten Meinungen zwischen Rat und Parlament vorzugehen. ${ }^{98}$

Auch im Hinblick auf die Haushaltsverantwortung der Kommission, wie diese durch Art. 317 AEUV festgelegt ist, scheint es kein normatives Hindernis für ein hier skizziertes Vorgehen zu geben. Diese Bestimmung verweist ausdrücklich auf den ,Grundsatz der Wirtschaftlichkeit der Haushaltsführung', welchem Art. 33 der EU-Haushaltsordnung ${ }^{99}$ bereits in seiner Überschrift als zentrale Eckpfeiler ,Wirtschaftlichkeit‘, ,Wirksamkeit' und ,Sparsamkeit‘ zuerkennt; letzterer Begriff sieht vor, „dass die Ressourcen, die von dem betreffenden Unionsorgan bei (...) Tätigkeiten eingesetzt werden, zum richtigen Zeitpunkt, in ausreichender Menge und angemessener Qualität sowie mit dem geringstmöglichen Kostenaufwand bereitgestellt werden"100. Die für den hiesigen Vorschlag angedachten Mittel würden dem Usus der Institutionen nicht widersprechen, da bereits die bis 2020 veranschlagten Ausgaben für die Regionalpolitik ,den größten Einzelposten'101 innerhalb des EU-Haushaltes und entsprechende Ausgaben mithin , das wichtigste Investitionsinstrument der Union' 102 darstellen: Am Ende könnte eine wirksame Behandlung, zumindest in Teilen, der Migrationsproblematik stehen.

Der hiesige Vorschlag soll sich speziell auf Personen ohne (oder mit rudimentär) dokumentierbare(r) Ausbildung beziehen, um diesen eine organisierte Aus-, gegebenenfalls Weiterbildung in praktischer Hinsicht (insbes. Handwerk und vergleichbare Dienstleistungen) zu ermöglichen, die auch dem Binnenmarkt insge-

96 Vgl. C. Waldhoff, in: Calliess/Ruffert (Fn. 21), Art. 314 AEUV, Rn. 15.

97 Vgl. Art. 17 Abs. 1 EUV.

98 Vgl. C. Waldhoff, in: Calliess/Ruffert (Fn. 21), Art. 314 AEUV, Rn. 15.

99 Verordnung (EU, Euratom) 2018/1046 des Europäischen Parlaments und des Rates vom 18. Juli 2018 über die Haushaltsordnung für den Gesamthaushaltsplan der Union, zur Änderung der Verordnungen (EU) Nr. 1296/2013, (EU) Nr. 1301/2013, (EU) Nr. 1303/2013, (EU) Nr. 1304/2013, (EU) Nr. 1309/2013, (EU) Nr. 1316/2013, (EU) Nr. 223/2014, (EU) Nr. 283/2014 und des Beschlusses Nr. 541/2014/EU sowie zur Aufhebung der Verordnung (EU, Euratom) Nr. 966/2012 (AB1. EU 2018 L 193/1).

100 Art. 33 Abs. 1 lit. a EU-Haushaltsordnung.

101 Europäische Kommission (Hrsg.), Die Europäische Union erklärt: Regionalpolitik (abrufbar unter Fn. 75), konkret S. 3.

102 Ibid. 
samt zugute käme. Mithin wären Personen die Zielgruppe, welche nicht durch insbes. die noch geltende Richtlinie 2009/50 hinsichtlich hochqualifizierter Beschäftigung ${ }^{103}$ abgedeckt sind - also Personen, welche eher über praktische Veranlagungen denn über Hochschulabschlüsse verfügen.

Die genannte Richtlinie, per se auf einen Personenkreis bestimmter Qualifikationen zugeschnitten, sieht als ein Essentialium ihrer Zulassungskriterien u.a. einen gültigen Arbeitsvertrag für die betreffende Person in einem Mitgliedstaat vor (Art. 5 Abs. 1 lit. a RL) ${ }^{104}$. Insoweit wird hier vorgeschlagen, dass die jeweilige Ratsvorsitz-Trias Sorge für die Erfassung entsprechender Bedürfnisse zunächst in ihren, darauffolgend auch in den anderen EU-Mitgliedstaaten trägt und entsprechende Aus- sowie Weiterbildungsmöglichkeiten und Arbeitsmarktschancen für jeden Mitgliedstaat bündelt, hierfür gleichsam eine finanzielle Förderung seitens der Union erhält.

Dies könnte aus Gründen der Rechtssicherheit freilich nur Migranten betreffen, deren Aufenthaltsstatus bereits geklärt ist, und sollte sich weitergehend nach den einschlägigen nationalen Bestimmungen (für die BRD insbes. die $\S \S 18 \mathrm{ff}$. AufenthG) richten. Insoweit wäre für die deutsche Rechtsordnung bereits auf $\S 18$ Abs. 1 AufenthG zu verweisen, wonach „(sich) die Zulassung ausländischer Beschäftigter (...) an den Erfordernissen des Wirtschaftsstandortes Deutschland unter Berücksichtigung der Verhältnisse auf dem Arbeitsmarkt und dem Erfordernis, die Arbeitslosigkeit wirksam zu bekämpfen (orientiert)“. Nicht zuletzt wäre dies auch europarechtlich gedeckt, da etwa die Richtlinie 2009/50 hinsichtlich hochqualifizierter Beschäftigung in ihrem Art. 3 Abs. 2 lit. a eine Anwendung bei nicht geklärter Rechtsstellung des betreffenden Drittstaatsangehörigen negiert. ${ }^{105}$

Ein Lösungsansatz wie vor geht bis dato ersichtlich nicht aus den Planungen der EU-Regionalpolitik, auch nicht bis 2020, hervor. ${ }^{106}$ Ebenso wenig kann solches dem ,Neuansiedlungsrahmen“ der EU entnommen werden, welcher von ,prioritären Regionen“ spricht. ${ }^{107}$ Es könnte dies gleichwohl idealerweise eine Art intensivierter Zusammenarbeit, eine Verständigung ,von unten nach oben“ zwischen den Regionen der Mitgliedstaaten, zwecks bestmöglichen Ausgleiches ihrer Bedürfnisse auf dem Arbeitsmarkt bilden. Evtl. wäre dies ein hilfreicher, da konstrukti-

103 Richtlinie 2009/50/EG des Rates vom 25. Mai 2009 über die Bedingungen für die Einreise und den Aufenthalt von Drittstaatsangehörigen zur Ausübung einer hochqualifizierten Beschäftigung (AB1. (EU) L 2009, 155/17). Der deutsche Gesetzgeber reagierte hierauf namentlich durch die Schaffung des § 19a AufenthG bzgl. der" Blaue(n) Karte EU". Zwischenzeitlich hat die Kommission einen Vorschlag zur Änderung dieser RL vorgelegt (Vorschlag für eine Richtlinie des Europäischen Parlaments und des Rates über die Bedingungen für die Einreise und für den Aufenthalt von Drittstaatsangehörigen zur Ausübung einer umfassende Qualifikationen voraussetzenden Beschäftigung vom 7. Juni 2016 (KOM/2016/0378 endg.): Gleichwohl hat auch dieser Vorschlag einen eingeschränkten Focus, da er zwecks Anwendung eben auch das Erfordernis eines "höheren beruflichen Bildungsabschluss(es)" (Art. 2 lit. b 3. Spiegelstrich i.V.m. Art. 2 litt. h und i des Vorschlages) vorsieht.

104 Vgl. ebenso auch Art. 5 Abs. 1 lit. b des RL-Vorschlages (KOM (2016) 378 endg.).

105 Vgl. ebenso auch Art. 3 Abs. 2 lit.b des RL-Vorschlages (KOM (2016) 378 endg.)

106 Vgl. Fn. 75.

107 Vgl. ,Eine umfassende Migrationssteuerung. Vermerk der Kommission zur Tagung des Europäischen Rates im Juni $2018^{\circ}$ (Fn. 67), insbes. S. 4/5. 
ver Beitrag zu einem frischen ,Narrativ` für die Fortentwicklung der EU, bestehenden Widrigkeiten zum Trotz. 